\title{
Fréquence de la consommation d'alcool chez les Canadiennes en âge de procréer
}

\author{
Mélanie Varin, M. Sc. (1); Elia Palladino, M. Sc. (1,2); Kate Hill MacEachern, Ph. D. (1); Lisa Belzak, M. Sc. S. (1); \\ Melissa M. Baker, Ph. D. (1)
}

Diffuser cet article sur Twitter

\section{Résumé}

Introduction. La publication de données sur la consommation d'alcool chez les Canadiennes en âge de procréer comble une lacune importante en matière de données probantes.

Méthodologie. Nous avons analysé, à l'aide des données de l'Enquête sur la santé dans les collectivités canadiennes de 2019, les taux de consommation hebdomadaire et de consommation excessive d'alcool chez les femmes de 15 à 54 ans en fonction de caractéristiques sociodémographiques, de la province de résidence et de la consommation simultanée d'autres substances.

Résultats. Parmi la population cible, 30,5 \% des femmes interrogées ont fait état d'une consommation hebdomadaire d'alcool et 18,3 \% d'une consommation excessive d'alcool au cours des 12 derniers mois. Les proportions variaient en fonction des caractéristiques sociodémographiques, de la province et de la consommation de substances. Les différences les plus notables et les plus significatives étaient liées à la consommation de cannabis et au tabagisme.

Conclusion. Ces renseignements peuvent aider les prestataires de soins de santé à évaluer la consommation d'alcool et à promouvoir une consommation d'alcool à faible risque afin de prévenir l'exposition à l'alcool durant la grossesse.

Mots-clés : consommation de substances, polyconsommation, femmes en âge de procréer, Directives de consommation d'alcool à faible risque du Canada

\section{Introduction}

L'alcool est largement consommé au Canada, plus de $75 \%$ de la population de 15 ans ou plus ayant déclaré en avoir consommé dans l'année ${ }^{1}$. De nombreuses conséquences négatives sont associées à la consommation d'alcool, communément appelées les méfaits de l'alcool. Il s'agit, entre autres, de blessures physiques ${ }^{2}$, d'effets néfastes sur la santé physique et mentale ${ }^{2,3}$, de cancers $^{4}$, de cirrhoses du foie ${ }^{5}$ et même de décès ${ }^{2,6,7}$.

Les Directives de consommation d'alcool à faible risque du Canada ont été publiées en 2011 afin de réduire les méfaits de l'alcool grâce à une meilleure sensibilisation de la population ${ }^{8}$. Ces directives décrivent les pratiques exemplaires qui se rapportent à la consommation sécuritaire d'alcool, en particulier la recommandation de s'abstenir de consommer de l'alcool pour les femmes qui planifient une grossesse ou qui sont enceintes ${ }^{8}$. Les stratégies de réduction des méfaits aident les cliniciens et les professionnels de la santé publique à promouvoir une consommation d'alcool à faible risque dans les catégories de population qui gagneraient à mieux connaître les risques pour la santé associés à la consommation d'alcool. De

\section{Points saillants}

- En 2019, 30,5 \% des femmes en âge de procréer ont fait état d'une consommation hebdomadaire d'alcool.

- En 2019, 18,3 \% des femmes en âge de procréer ont fait état d'une consommation d'alcool excessive, supérieure aux directives de consommation d'alcool à faible risque du Canada.

- Ces proportions varient selon les caractéristiques sociodémographiques, la province de résidence, le tabagisme et la consommation de cannabis.

- Les taux estimés de consommation hebdomadaire ou excessive d'alcool étaient entre deux et trois fois plus élevés chez les femmes ayant déclaré être fumeuses, ex-fumeuses ou fumeuses à titre expérimental ou ayant fait état d'une consommation de cannabis au cours de l’année écoulée.

telles stratégies sont particulièrement importantes pour les femmes en âge de procréer, étant donné qu'elles peuvent tomber enceintes, que ce soit de façon planifiée ou non, pendant cette période de leur vie et que la consommation d'alcool pendant la grossesse peut avoir des répercussions importantes sur le fœtus. En 2017, 4,2 \% des Canadiennes ayant accouché au cours des 5 années précédentes avaient déclaré avoir consommé de l'alcool durant leur grossesse?

Dans cette étude, 1) nous rendons compte des taux de consommation hebdomadaire 
et de consommation excessive d'alcool chez les femmes de 15 à 54 ans au Canada et 2) nous analysons la consommation d'alcool dans cette population en fonction des caractéristiques sociodémographiques, de la province de résidence, du tabagisme et de la consommation de cannabis. Étant donné qu'à notre connaissance, il n'existe pas d'estimations nationales antérieures ou actuelles de la consommation d'alcool des femmes en âge de procréer, nous cherchons à combler cette lacune.

\section{Méthodologie}

À l'aide des données du cycle annuel de 2019 (janvier à décembre) de l'Enquête sur la santé dans les collectivités canadiennes (ESCC), nous avons évalué deux comportements de consommation d'alcool au sein de la population du Canada pour les répondantes s'étant déclarées de sexe féminin et âgées de 15 à 54 ans : la consommation hebdomadaire d'alcool et la consommation excessive d'alcool. L'ESCC est une enquête nationale transversale menée par Statistique Canada qui fournit de l'information sur la santé. L'ESCC de 2019 excluait les femmes vivant dans les territoires, dans les réserves des Premières Nations ou dans d'autres établissements autochtones des provinces, ainsi que les membres à temps plein des Forces armées canadiennes et les personnes vivant en établissement.

De plus amples détails sur la conception et le cadre d'échantillonnage de l'ESCC sont accessibles sur le site Internet de Statistique Canada ${ }^{1}$.

\section{Consommation hebdomadaire d'alcool}

On a demandé aux répondantes : " $\mathrm{Au}$ cours des 12 derniers mois, à quelle fréquence avez-vous consommé des boissons alcoolisées? ». Les options de réponse étaient : " moins d'une fois par mois ", " une fois par mois », " 2 à 3 fois par mois ", " une fois par semaine ", " 2 à 3 fois par semaine », " 4 à 6 fois par semaine » et " tous les jours ». On a défini la consommation hebdomadaire d'alcool comme correspondant aux réponses « une fois par semaine ", " 2 à 3 fois par semaine ", " 4 à 6 fois par semaine » et " tous les jours ». Nous avons exclu de l'analyse les femmes en âge de procréer auxquelles le code " ne sait pas ", "refus " ou « non déclaré » avait été attribué $(\mathrm{n}=72)$.

\section{Consommation excessive d'alcool}

On a demandé aux répondantes : " $\mathrm{Au}$ cours des 12 derniers mois, combien de fois avez-vous bu quatre verres d'alcool ou plus à une même occasion? ». Les options de réponse étaient : " jamais ", " moins d'une fois par mois ", " une fois par mois ", " 2 à 3 fois par mois », " une fois par semaine » et «plus d'une fois par semaine ». On a défini la consommation excessive d'alcool comme correspondant aux réponses " une fois par mois ", " 2 à 3 fois par mois », " une fois par semaine » et "plus d'une fois par semaine $»^{10}$. Nous avons exclu de l'analyse les femmes en âge de procréer auxquelles le code " ne sait pas ", " refus » ou " non déclaré » avait été attribué $(\mathrm{n}=108)$.

Nous avons estimé les taux pondérés de consommation hebdomadaire et de consommation excessive d'alcool dans la population cible, avec des intervalles de confiance à $95 \%$. Nous avons utilisé comme numérateur le nombre total de femmes en âge de procréer ayant fait état d'une consommation hebdomadaire ou excessive d'alcool et comme dénominateur le nombre total de femmes en âge de procréer ayant répondu à la question.

Nous avons ventilé les estimations par groupe d'âge, quintile de revenu du ménage, province de résidence, état matrimonial, résidence en milieu urbain ou rural, statut d'immigrante, emploi au cours des 12 derniers mois, statut d'étudiante, consommation de cannabis et tabagisme au cours des 12 derniers mois. Les taux estimés pondérés ont été calculés à l'aide des poids d'échantillonnage fournis par Statistique Canada. La variance a été estimée par la méthode bootstrap, et la version 7.1 du logiciel SAS Enterprise Guide (SAS Institute Inc., Cary, Caroline du Nord, États-Unis) a été utilisée pour les analyses statistiques. Nous avons établi la signification statistique d'après le chevauchement des intervalles de confiance.

\section{Résultats}

Dans l'ensemble, 30,5 \% des femmes en âge de procréer ont déclaré avoir consommé de l'alcool sur une base hebdomadaire au cours de l'année écoulée, et $18,3 \%$ ont déclaré avoir une consommation excessive d'alcool. Les valeurs estimées varient lorsque les données sont désagrégées (tableau 1). Les différences statistiquement significatives sont détaillées dans les sections suivantes.

\section{Consommation hebdomadaire d'alcool}

Les taux de consommation hebdomadaire d'alcool (" consommation régulière ») augmentaient avec l'âge (de $7,1 \%$ à $35,6 \%$ ) et le revenu (de 19,8 \% à 41,2\%). La plus forte prévalence de consommation hebdomadaire d'alcool dans la population cible était au Québec (41,0\%) et la plus faible $(20,0 \%)$ au Nouveau-Brunswick. Parmi les femmes en âge de procréer ayant fait état d'une consommation hebdomadaire d'alcool, il y en avait davantage qui étaient mariées ou en union libre (33,7\%) que veuves, séparées, divorcées, célibataires ou jamais mariées (26,3\%).

Les non-immigrantes $(37,6 \%)$ et les nonétudiantes $(33,4 \%)$ affichaient des taux de consommation hebdomadaire d'alcool plus élevés que les immigrantes $(13,6 \%)$ et les étudiantes (19,1 \%). Parmi les femmes en âge de procréer ayant fait état d'une consommation hebdomadaire d'alcool, le pourcentage de celles qui avaient occupé un emploi ou travaillé dans une entreprise au cours de l'année écoulée $(33,9 \%)$ était plus élevé que le pourcentage de celles n'ayant pas occupé d'emploi ni travaillé dans une entreprise $(13,9 \%)$.

La consommation hebdomadaire d'alcool était environ deux fois plus fréquente chez les fumeuses $(40,3 \%)$, les exfumeuses $(47,0 \%)$ et les fumeuses à titre expérimental $(43,2 \%)$ que chez les abstinentes à vie $(21,6 \%)$. Les femmes en âge de procréer ayant consommé du cannabis au cours des 12 derniers mois ont fait état d'une consommation hebdomadaire d'alcool presque deux fois supérieure à celle des femmes n'ayant pas consommé de cannabis au cours des 12 derniers mois (46,0\% contre $26,3 \%$ ).

\section{Consommation excessive d'alcool}

Les femmes âgées de 20 à 29 ans étaient celles qui présentaient le plus haut taux de consommation excessive d'alcool (25,2 \%). Les taux de consommation excessive d'alcool augmentaient avec le revenu, se situant entre $15,4 \%$ et $23,7 \%$ selon le quintile de revenu. La consommation excessive d'alcool chez les femmes en âge de procréer atteignait sa valeur maximale à Terre-Neuve-et-Labrador $(26,6 \%)$ et sa valeur minimale au Nouveau-Brunswick $(14,6 \%)$. Parmi les femmes en âge de 
TABLEAU 1

Taux estimés de consommation hebdomadaire et de consommation excessive d'alcool au cours de la dernière année chez les femmes en âge de procréer, selon des caractéristiques sociodémographiques et autres, Canada et par province, 2019

\begin{tabular}{|c|c|c|c|}
\hline \multirow[b]{2}{*}{ Variables } & \multicolumn{3}{|c|}{ Taux (IC à $95 \%$ ) } \\
\hline & $\begin{array}{l}\text { Proportion dans } \\
\text { l'échantillon (\%) }\end{array}$ & $\begin{array}{l}\text { Proportion ayant fait état d'une } \\
\text { consommation hebdomadaire } \\
\text { d'alcool (\%) }\end{array}$ & $\begin{array}{l}\text { Proportion ayant fait état d'une } \\
\text { consommation excessive d'alcool au } \\
\text { moins une fois par mois (\%) }\end{array}$ \\
\hline 15 à 19 & $9,8$ (9,1 à 10,6$)$ & 7,1 (5,1 à 9,0) & $10,6(8,1$ à 13,1$)$ \\
\hline 20 à 29 & $23,9(22,9$ à 24,9$)$ & 29,8 (27,1 à 32,5) & $25,2(22,8$ à 27,7$)$ \\
\hline 40 à 54 & 38,5 (37,6 à 39,3) & 35,6 (33,6 à 37,5) & 17,2 (15,7 à 18,7) \\
\hline \multicolumn{4}{|l|}{ Revenus } \\
\hline Q1 (revenu le plus faible) & $21,8(20,7$ à 23,0$)$ & $19,8$ (17,3 à 22,3$)$ & $15,4(13,1$ à 17,7$)$ \\
\hline Q2 & $19,2(18,1$ à 20,3$)$ & $27,1(24,3$ à 29,8$)$ & $17,3(15,1$ à 19,6$)$ \\
\hline Q3 & $20,1(19,0$ à 21,3$)$ & 30,6 (27,9 à 33,2) & $16,5(14,4$ à 18,5$)$ \\
\hline Colombie-Britannique & $12,9(12,7$ à 13,2$)$ & 29,9 (26,6 à 33,2) & $20,5(17,5$ à 23,4$)$ \\
\hline Alberta & 12,4 (12,1 à 12,6) & $31,8(28,4$ à 35,3) & $18,0(15,2$ à 20,9$)$ \\
\hline Saskatchewan & 2,9 (2,8 à 3,0) & 27,9 (22,3 à 33,6) & $17,1(12,6$ à 21,5$)$ \\
\hline Manitoba & 3,5 (3,4 à 3,6) & $30,1(24,8$ à 35,4$)$ & 20,4 (15,6 à 25,2) \\
\hline Ontario & $40,6(40,1$ à 41,0$)$ & 25,5 (23,6 à 27,5) & $15,5(13,8$ à 17,2$)$ \\
\hline Québec & $21,7(21,4$ à 22,1$)$ & 41,0 (38,3 à 43,7) & $21,6(19,4$ à 23,8) \\
\hline Nouveau-Brunswick & 1,9 (1,8 à 1,9) & $20,0(14,8$ à 25,3$)$ & $14,6^{C}(10,1$ à 19,1$)$ \\
\hline Nouvelle-Écosse & 2,5 (2,4 à 2,6) & $34,1(29,0$ à 39,2$)$ & $23,1(18,4$ à 27,8$)$ \\
\hline Île-du-Prince-Édouard & $0,4(0,4$ à 0,4$)$ & $23,9^{c}(16,7$ à 31,1$)$ & $19,4^{\mathrm{C}}(13,2$ à 25,6$)$ \\
\hline Urbain & $85,5(84,7$ à 86,2$)$ & 30,1 (28,7 à 31,4) & $17,6$ (16,5 à 18,7$)$ \\
\hline Rural & $14,5$ (13,8 à 15,3$)$ & 33,0 (30,6 à 35,5) & $22,4(20,3$ à 24,6$)$ \\
\hline \multicolumn{4}{|l|}{ Statut d'immigrante } \\
\hline Oui & $29,8(28,4$ à 31,1$)$ & 13,6 (11,9 à 15,3) & 6,9 (5,7 à 8,2) \\
\hline Non & 70,2 (68,9 à 71,6) & 37,6 (36,2 à 39,0) & $23,1(21,8$ à 24,3$)$ \\
\hline \multicolumn{4}{|c|}{ A occupé un emploi rémunéré ou travaillé dans une entreprise au cours des 12 derniers mois } \\
\hline Oui & $83,2(82,2$ à 84,3$)$ & $33,9(32,6$ à 35,3$)$ & $20,4(19,2$ à 21,5$)$ \\
\hline Non & $16,8(15,7$ à 17,8$)$ & $13,9(11,7$ à 16,2$)$ & 8,2 (6,5 à 9,9) \\
\hline \multicolumn{4}{|l|}{ Étudiante } \\
\hline Oui & $20,2(19,2$ à 21,3$)$ & $19,1(16,7$ à 21,5$)$ & $16,6(14,3$ à 18,8$)$ \\
\hline Non & $79,8(78,7$ à 80,8$)$ & $33,4(32,0$ à 34,8$)$ & $18,8(17,6$ à 19,9$)$ \\
\hline \multicolumn{4}{|l|}{ Tabagisme } \\
\hline Abstinente à vie & $59,4(58,0$ à 60,7$)$ & $21,6(20,2$ à 23,1$)$ & $11,3(10,2$ à 12,4$)$ \\
\hline Fumeuse & $13,4(12,6$ à 14,2$)$ & 40,3 (37,2 à 43,4) & 35,4 (32,3 à 38,5) \\
\hline Ex-fumeuse & $14,0(13,1$ à 14,9$)$ & 47,0 (43,9 à 50,2) & 27,3 (24,5 à 30,0) \\
\hline \multirow[t]{2}{*}{ Fumeuse à titre expérimental } & $13,2(12,3$ à 14,2$)$ & 43,2 (39,4 à 46,9) & 23,0 (19,9 à 26,2) \\
\hline & & & Suite à la page suiva \\
\hline
\end{tabular}


TABLEAU 1 (suite)

Taux estimés de consommation hebdomadaire et de consommation excessive d'alcool au cours de la dernière année chez les femmes en âge de procréer, selon des caractéristiques sociodémographiques et autres, Canada et par province, 2019

\begin{tabular}{|c|c|c|c|}
\hline \multirow[b]{2}{*}{ Variables } & \multicolumn{3}{|c|}{ Taux (IC à $95 \%$ ) } \\
\hline & $\begin{array}{l}\text { Proportion dans } \\
\text { l'échantillon (\%) }\end{array}$ & $\begin{array}{c}\text { Proportion ayant fait état d'une } \\
\text { consommation hebdomadaire } \\
\text { d'alcool (\%) }\end{array}$ & $\begin{array}{l}\text { Proportion ayant fait état d'une } \\
\text { consommation excessive d'alcool au } \\
\text { moins une fois par mois (\%) }\end{array}$ \\
\hline \multicolumn{4}{|c|}{ Consommation de cannabis au cours des 12 derniers mois } \\
\hline Oui & $23,0(21,9$ à 24,2$)$ & $46,0(43,2$ à 48,7$)$ & $39,1(36,4$ à 41,8$)$ \\
\hline Non & 77,0 (75,8 à 78,1) & $26,3(25,0$ à 27,6$)$ & $12,4(11,5$ à 13,4$)$ \\
\hline
\end{tabular}

Source des données : Enquête sur la santé dans les collectivités canadiennes (ESCC) de 2019.

Abréviation : IC, intervalle de confiance.

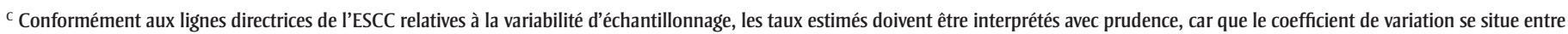
$15,1 \%$ et $25,0 \%$.

procréer, davantage de femmes veuves, séparées, divorcées, célibataires ou jamais mariées $(21,1 \%)$ ont déclaré une consommation excessive d'alcool que de femmes mariées ou en union libre (16,3\%).

Le pourcentage de femmes en âge de procréer déclarant une consommation excessive d'alcool était supérieur chez celles vivant en milieu rural $(22,4 \%)$ que chez celles vivant en milieu urbain (17,6\%). Une plus grande proportion de femmes ont fait état d'une consommation excessive d'alcool chez les non-immigrantes $(23,1 \%)$ par rapport aux immigrantes $(6,9 \%)$ et chez celles ayant exercé un emploi rémunéré au cours des 12 derniers mois (20,4 \%) par rapport à celles n'ayant pas exercé d'emploi rémunéré au cours de l'année écoulée $(8,2 \%)$.

Les taux de consommation excessive d'alcool chez les fumeuses $(35,4 \%)$, les ex-fumeuses $(27,3 \%)$ et les fumeuses à titre expérimental $(23,0 \%)$ variaient entre le double et le triple de ceux observés chez les abstinentes à vie (11,3\%). Les femmes en âge de procréer ayant déclaré avoir consommé du cannabis au cours des 12 derniers mois $(39,1 \%)$ avaient un taux de consommation excessive d'alcool plus de trois fois supérieur à celui de celles n'ayant pas consommé de cannabis au cours des 12 derniers mois $(12,4 \%)$.

\section{Analyse}

En 2019, 30,5 \% des femmes en âge de procréer ont déclaré avoir consommé de l'alcool sur une base hebdomadaire, et $18,3 \%$ ont fait état d'une consommation excessive d'alcool. Bien qu'il existe de légères différences dans les définitions de la consommation excessive d'alcool (avoir bu quatre verres ou plus en une même occasion au moins une fois par mois au cours de l'année écoulée) et d'excès occasionnels d'alcool (avoir bu quatre verres ou plus en une même occasion au moins une fois au cours de l'année écoulée), la proportion de consommation excessive d'alcool est similaire à la fréquence médiane d'excès occasionnels d'alcool en 2019 $(19,2 \%)$ chez les femmes de 18 à 44 ans aux États-Unis ${ }^{11}$.

Nous avons constaté que la consommation hebdomadaire ou excessive d'alcool était en proportion la plus forte chez les femmes en âge de procréer appartenant au quintile de revenu le plus élevé, n'étant pas immigrantes, ayant occupé un emploi au cours des 12 derniers mois et n'étant pas étudiantes. Nous avons également constaté une hétérogénéité entre les comportements de consommation d'alcool autodéclarés. Par exemple, si la consommation hebdomadaire d'alcool était plus répandue chez les femmes en âge de procréer mariées ou en union libre que chez celles qui étaient veuves, séparées, divorcées, célibataires ou qui n'avaient jamais été mariées, cette tendance était inversée pour les comportements de consommation excessive d'alcool. De même, le taux de consommation hebdomadaire d'alcool le plus élevé était celui des femmes de 40 à 49 ans, tandis que le taux de consommation excessive d'alcool était le plus élevé dans la tranche d'âge des 20 à 29 ans. Étant donné ces différences, les études futures devraient comparer plusieurs comportements de consommation selon divers déterminants sociaux de la santé, dans l'objectif de mieux comprendre la consommation d'alcool dans cette population.
Nos résultats les plus remarquables concernent l'usage du tabac et la consommation de cannabis, un indicateur possible de polyconsommation. Les taux de consommation hebdomadaire ou excessive d'alcool au cours de l'année écoulée étaient entre deux et trois fois supérieurs chez les femmes qui fumaient, avaient fumé ou fumaient à titre expérimental ou qui avaient déclaré consommer du cannabis. Ces données peuvent aider les prestataires de soins de santé à évaluer la polyconsommation au sein de cette population et à promouvoir une consommation d'alcool à faible risque comme mesure de prévention de l'exposition à l'alcool pendant la grossesse.

\section{Points forts et limites}

Notre étude fournit pour la première fois des estimations représentatives à l'échelle nationale quant à la fréquence de deux comportements de consommation d'alcool chez les femmes en âge de procréer, et ce, en fonction de diverses variables sociodémographiques et de la consommation de substances. Bien que nos résultats comblent une lacune en matière de données probantes, nous avons quelques limites à mentionner.

Premièrement, comme les données sont transversales, nous ne pouvons pas établir de temporalité. Deuxièmement, toutes les données de l'analyse étaient autodéclarées et donc sujettes aux biais de déclaration et de désirabilité sociale. Enfin, en raison des critères d'exclusion de l'ESCC, nous n'avons pas été en mesure d'inclure de données sur les femmes en âge de procréer vivant dans les territoires ou dans les réserves des Premières Nations. 


\section{Recherches supplémentaires}

Pour déterminer à quels groupes de femmes en âge de procréer une meilleure connaissance des directives de consommation d'alcool à faible risque serait bénéfique, les recherches futures devraient examiner les associations entre caractéristiques sociodémographiques, consommation d'autres substances et consommation d'alcool.

\section{Remerciements}

Les auteures tiennent à remercier leurs collègues de la Division de la santé de la mère, de l'enfant et des jeunes qui les ont invitées à collaborer et à soumettre conjointement des articles de type aperçu. Nous tenons également à remercier Adam Probert, Claudia Lagacé et Sarah Palmeter pour leur aide dans l'interprétation des résultats et la révision de cet aperçu.

\section{Conflits d'intérêts}

Aucun.

\section{Contribution des auteures et avis}

Toutes les auteures ont donné leur avis sur la conception et l'élaboration de l'analyse des données secondaires. MV a rédigé cet aperçu et a effectué l'analyse descriptive. Toutes les coauteures ont interprété les données et revu d'un œil critique ou commenté chacune des ébauches du manuscrit.

Le contenu de l'article et les points de vue qui y sont exprimés n'engagent que les auteures; ils ne correspondent pas nécessairement à ceux du gouvernement du Canada.

\section{Références}

1. Statistique Canada. Enquête sur la santé dans les collectivités canadiennes - Composante annuelle (ESCC) : Information détaillée pour 2019 [Internet]. Ottawa (Ont.) : Statistique Canada [modification le 18 novembre 2019; consultation le 16 novembre 2020]. En ligne à : https://www23.statcan .gc.ca/imdb/p2SV_f.pl?Function $=$ getSurvey\&Id $=1208978$

2. Shield KD, Kehoe T, Taylor B, Patra J, Rehm J. Alcohol-attributable burden of disease and injury in Canada, 2004. Int J Public Health. 2012;57(2): 391-401. https://doi.org/10.1007/s00038 $-011-0247-7$
3. Iranpour A, Nakhaee N. A review of alcohol-related harms: a recent update. Addict Health. 2019;11(2):129-137. https://doi.org/10.22122/ahj.v11i2.225

4. The Lancet. Alcohol and cancer. Lancet. 2017;390(10109):2215. https://doi.org /10.1016/S0140-6736(17)32868-4

5. Roerecke M, Vafaei A, Hasan OS, et al. Alcohol consumption and risk of liver cirrhosis: a systematic review and meta-analysis. Am J Gastroenterol. 2019;114(10):1574-1586. https://doi .org/10.14309/ajg.0000000000000340

6. Orpana H, Giesbrecht N, Hajee A, Kaplan MS. Alcohol and other drugs in suicide in Canada: opportunities to support prevention through enhanced monitoring. Inj Prev. 2021;27(2):194200. https://doi.org/10.1136/injuryprev $-2019-043504$

7. Ramstedt M. Alcohol consumption and alcohol-related mortality in Canada, 1950-2000. Can J Public Health. 2004; 95(2):121-126. https://doi.org/10.1007 /BF03405779

8. Comité consultatif sur la Stratégie nationale sur l'alcool. Directives de consommation d'alcool à faible risque du Canada. Ottawa (Ont.) : Centre canadien sur les dépendances et l'usage de substances; 2018.

9. Centre de surveillance et de recherche appliquée. Indicateurs de la santé périnatale (ISP). Infobase de la santé publique [Internet]. Ottawa (Ont.) : Agence de la santé publique du Canada; [modification le 20 octobre 2020; consultation le 16 avril 2021]. En ligne à : https://sante-infobase. canada.ca/isp/outil-de-donnees $/$ ?Dom $=1 \&$ Ind $=1 \&$ MS $=1 \&$ Strat $=1$

10. Statistique Canada. Feuillets d'information de la santé : consommation abusive d'alcool, 2018 [Internet]. Ottawa (Ont.) : Statistique Canada [modification le 25 juin 2020; consultation le 16 novembre 2020]. En ligne à : https://www150.statcan.gc.ca/n1/pub /82-625-x/2019001/article/00007-fra.htm

11. National Center on Birth Defects and Developmental Disabilities. Fetal alcohol spectrum disorders (FASDs): Statelevel estimates of alcohol use among women - 2019 [Internet]. Atlanta (GA) : Centers for Disease Control and Prevention [modification le 14 mai 2021; consultation le 27 mai 2021]. En ligne à : https://www.cdc.gov/ncbddd /fasd/data-maps-2019.html 